\title{
La construcción del prestigio literario en Colombia. El caso de Mario Mendoza, Jorge Franco y Santiago Gamboa
}

\author{
Margarita Valencia ${ }^{1}$
}

Resumen. Esta investigación pretende caracterizar las trayectorias editoriales de Mario Mendoza, Jorge Franco, Santiago Gamboa y determinar las fuentes del prestigio literario entre los narradores colombianos contemporáneos. ¿Qué relación hay entre la construcción del prestigio literario y los sellos editoriales que publican sus obras? Se busca analizar el lugar que ocupan otras fuentes de prestigio literario en las trayectorias literarias de los autores citados: premios, circulación, difusión en la prensa, trayectorias académicas.

Palabras clave: Prestigio literario; literatura colombiana contemporánea.

[en] The Construction of Literary Prestige in Colombia. The Case of Mario Mendoza, Jorge Franco and Santiago Gamboa

\begin{abstract}
This research into the publishing history of Colombian authors Mario Mendoza, Jorge Franco and Santiago Gamboa is aimed at discovering the sources of literary prestige in the case of Colombian contemporary authors. What's the relation between their publishers and their literary prestige? Other sources of literary prestige, such as awards, translations, press coverage, academic experience.

Keywords: Lliterary prestige; Colombian contemporary literature.

Sumario: 1. El prestigio. 2. Elementos que contribuyen a la creación del prestigio. 3. Circunstancias particulares del ámbito editorial. 4. Franco, Mendoza y Gamboa. 5. La construcción del prestigio. 5.1. Las editoriales. 5.2. Los premios. 5.3. La prensa. 5.4. La academia. 6. Conclusiones. 7. Anexo.
\end{abstract}

Cómo citar: Valencia, M. (2020) La construcción del prestigio literario en Colombia. El caso de Mario Mendoza, Jorge Franco y Santiago Gamboa, en Anales de Literatua Hispanoamericana 49, 255-267.

\section{El prestigio}

Una confrontación entre la definición de prestigio del DRAE — "Pública estima de alguien o de algo, fruto de su mérito"- y la de dictionary.com - "widespread respect and admiration felt for someone or something on the basis of a perception of their achievements or quality" - nos permite entrar rápidamente en materia. Mientras que la definición del diccionario español se refiere al mérito de quien ostenta el prestigio, y establece que este se manifiesta en la esfera de lo público, la definición inglesa se refiere a la percepción de quienes adscriben el prestigio. Aquel prestigio parece brotar en el vacío mientras que este nace en un contexto social. Esa diferencia es fundamental a la hora de definir qué entendemos hoy por prestigio, y su diferencia con la fama o la calidad literaria.

La fama ha estado siempre asociada a la literatura. Los héroes homéricos (Curtius, 476) reconocían el papel esencial de la poesía en la consecución de la fama eterna y sabían que las obras de los poetas eran el camino a la inmortalidad. Pero fue Quinto Horacio Flaco quien incluyó al poeta en el circuito en el que antes solo participaban el héroe y el poema y fue él quien estableció los parámetros para una teoría de la fama literaria. Gran parte de las expresiones relacionadas con la fama literaria nacieron en sus obras $(\mathrm{H}$. J. Jackson, 8) y el poeta romano se vanagloriaba del reconocimiento de la gente en la calle. Si duda los 
términos de esta asociación han cambiado, pero reconocemos aún el deseo de fama e inmortalidad que impulsa a los hombres.

La longevidad de la obra literaria, el paso de un texto de una generación a otra, es la pregunta fundamental en la conformación del canon (Underwood y Sellers, 321), y este es uno de los tópicos esenciales de la teoría literaria. Pero la consagración de la obra no está necesariamente relacionada con el prestigio del autor, un elemento relativamente nuevo en el universo de la creación literaria que tiene más que ver con las percepciones de los actores en el campo que con la calidad literaria. Verboord (62) nos advierte:

A classification by 'literary prestige' is preferable to a classification by 'literary quality'. Using the term quality suggests that it is possible to establish the intrinsic literary value of texts, while using the term 'prestige' implies that the classification of authors in the literary field is a socially determined process.

Nos interesa aquí este proceso social al que se somete a los autores literarios, esta trayectoria que, de acuerdo con Dubois (2014), pasa por cuatro momentos: emergencia, reconocimiento, consagración y canonización. Y el paso de un estadio a otro suele estar relacionado con un sello editorial (o un editor) pues, como nos lo recuerda Bourdieu (13), los artistas pudieron reafirmar plenamente su pretensión a la consagración sólo cuando los editores reemplazaron a los mecenas (que esperaban la subordinación del artista a su propio ideal cultural). De allí que se relacione el prestigio de un autor con su asociación con un editor, cuando este es un agente cultural reconocido.

\section{Elementos que contribuyen a la creación del prestigio}

El reconocimiento suele estar relacionado con la riqueza, con los bienes materiales, y por tanto con las cifras. En el caso del sistema literario, la cantidad de ejemplares vendidos (o la cantidad de ediciones) es un buen indicador del éxito de un autor. Pero "la competencia por la legitimidad intelectual nunca se identifica completamente con la competencia por el éxito en el mercado" (Bourdieu, 14). Seguimos adhiriéndonos a la idea de que el valor estético es irreductible al valor económico, de manera que el público anónimo le da a la obra una sanción económica, pero la relación ambivalente que los escritores tienen con el gran público (Bourdieu, 94) hace que la popularidad y la legitimidad cultural no siempre vayan de la mano.

Más que el público, parecería que son unos cuantos lectores los encargados de cimentar el prestigio de un escritor. "No se trata de cuántos te leen sino de quién te lee", dice Tom Vanderbilt en un ensayo en la revista The New Yorker (epítome, en la escena literaria neoyorquina, del experto cultural legitimador). Suele ser una verdad de perogrullo que los elementos que apuntalan la popularidad escolar no sirven para predecir el éxito en la vida real, añade. "Lo que explica que un libro se convierta en un éxito monumental suele tener poco que ver con su éxito posterior."

Este pequeño grupo de lectores está formado por críticos literarios, periodistas, profesores universitarios. $\mathrm{Y}$ otros escritores. Una de las funciones de la sociedad de los artistas es ser ella misma su propio mercado (Bourdieu, 26); en el caso del sistema literario, es fundamental la lectura de los pares o de escritores consagrados que se encarguen de apreciar inmediatamente las obras y darlas a conocer al público. Cuando los expertos culturales institucionalmente legitimados atribuyen al autor y/o a la obra un cierto valor y manifiestan su admiración, abren el camino a la consagración cultural Veerbord $(2011,293)$.

Las editoriales también son importantes en el proceso de la creación de prestigio, en parte porque la acumulación de prestigio por parte del sello es un capital simbólico que el autor puede compartir. El sello busca que los consumidores lo identifiquen con unos criterios de selección determinados. En su estudio sobre el comportamiento de las editoriales, De Glas (1998) plantea la hipótesis de que las editoriales no son solo guardianes (gate-keepers) sino que juegan un papel fundamental en el desarrollo de la obra literaria de un autor:

The publishing house determines "who is part of the scene, who can call themselves a writer"; the publishing house regulates the appearance of works in the market, coaches the author decides who will continue to be published.

Es también relevante el hecho de que para muchos actores en el sector cultural sigue siendo válida la distinción entre los editores que son solo empresarios y cuyo objetivo es acomodarse a una demanda para maximizar sus ganancias en el corto plazo y aquellos cuyo interés principal es cultural. Danny Anderson (5) 
matiza esta diferencia en su análisis de la editorial Joaquín Mortiz como creadora de prestigio literario, y su papel en el reconocimiento logrado por los jóvenes autores mexicanos de los sesenta. "Publicar en Joaquín Mortiz era una meta que valía la pena", afirma el editor José Agustín, pero esta es una afirmación que debe matizarse: "Somewhere between the idealized view of the editor as a beneficient cultural Maecenas and the humoristic view of the editor as a consumate opportunist lies a more appropiate middle ground" (Anderson 5) Habría que decir también que este punto medio del cual habla Anderson está siendo ocupado cada vez más por la figura del agente, que exime al escritor del escarnio público de tomar decisiones exclusivamente económicas.

Quizás la fuente de prestigio más tradicional y más cuestionada hoy son los premios literarios. En un artículo de comienzos de 2017, Maribel Marín aborda el problema desde todas las perspectivas:

El Planeta (1952), el Biblioteca Breve (1958), el Alfaguara (1965), el Anagrama (1973), el Herralde (1983), el Tusquets (2005)..., unos con un perfil más comercial, otros más literario, unos con vocación más española, otros más latinoamericana, han contribuido también, con interrupciones, a revelar o consolidar autores, a crear lectores, y a construir un sistema de premios sin parangón, a medio camino entre el arte, el dinero y la vida social.

Pero este sistema de premios sin parangón no ha resistido el embate de quienes insisten en la defensa de la calidad literaria, y la seguidilla de escándalos ha acabado por lograr que el público asocie los premios con una operación exclusivamente comercial:

La desenfrenada escalada que impulsó ha llevado al premio hasta los 601.000 euros que se embolsa hoy - antes de impuestos - el ganador del Planeta, muy por delante de los 175.000 dólares (164.000 euros) del Alfaguara, los 125.000 euros del RBA de Novela Negra, los 100.000 del Primavera de Novela, los 30.000 del Biblioteca Breve... Y esa fuerte inversión que realizan las editoriales, a la que hay que añadir, en algunos casos, el premio a los finalistas además de las giras de los premiados — que por ejemplo a Alfaguara le suponen más de 100.000 euros más—, los actos de entrega de los galardones, las invitaciones a periodistas... tenía y tiene una contrapartida. Dado que un libro raramente supera los dos años de vida, exige recuperar rápido la inversión, lo que, en ocasiones, lleva a las empresas, como apunta González-Ariza refiriéndose al Planeta, "no a buscar grandes novelas", sino novelas "rentables", premios para un público mayoritario que, si alguna vez hicieron de brújula, hoy es dudoso que lo hagan. "En ningún caso son una guía literaria. Incluso es posible que sean todo lo contrario", dice Caballero Bonald. La agente literaria Antonia Kerrigan sí concede ese papel "al Premio Anagrama, que toma riesgos y busca talentos, y el Alfaguara, con la difusión de latinoamericanos desconocidos en España".

La polémica en la que se han visto envueltos los premios españoles refleja, por supuesto, la persistencia en el ámbito editorial de la confrontación entre la alta cultura y la cultura popular, entre lo comercial y lo cultural, que se vive con particular agudeza en América Latina.

\section{Circunstancias particulares del ámbito editorial}

Antes de 1936, la edición en castellano para España y América Latina estaba dominada por el sector editorial español; pero a partir de la Guerra Civil, Argentina y México asumieron el liderazgo y lo conservaron hasta bien entrados la década de los cincuenta, cuando la recuperación económica de España le permitió volver a la competencia. A lo largo de la segunda mitad del siglo 20, las editoriales españolas empezaron a abrir filiales en América Latina cuyo propósito fundamental era la comercialización de libros producidos en España. Sin embargo en los ochenta y noventa hubo un cambio de estrategia y empezaron a editar de forma masiva autores latinoamericanos y a absorber editoriales históricas latinoamericanas (Fernández, 2009), de manera que las filiales se convirtieron en "empresas plenamente adaptadas a la demanda de cada país. Las editoriales cuentan con un catálogo troncal al que añaden una serie de títulos propios del país en el que operan, con autores y temas locales".

La consecuencia más inmediata de esta estrategia en el sector literario latinoamericano fue la desaparición de las redes culturales y comerciales, formales o informales, que se habían establecido en el continente, y la balcanización de la producción: los autores latinoamericanos editados localmente por las editoriales españolas raramente se internacionalizaban, incluso en América Latina. 
En 2015 Cristóbal Pera (76), que en ese momento era director editorial de Penguin Random House en México, explicó así el cambio en el sector editorial latinoamericano:

There was a time when Latin America was the end destination for the remainders from the big publishers based in Spain. Mexican branches would rely mostly on the lists coming from the editorial programs in Spain. That began to change more than a decade ago, but now, with a drop of $35 \%$ in sales in the Spanish market, the American counterparts are taking a more protagonist role. As I mentioned before, $50 \%$ of the titles of our list are from rights acquired by Spain and $50 \%$ are from local authors.

A la caída del mercado español hay que añadir el crecimiento y la profesionalización del sector literario latinoamericano —crecimiento ligado, naturalmente, al crecimiento del público lector-. Las carreras literarias y editoriales de los escritores colombianos Jorge Franco, Mario Mendoza y Santiago Gamboa reflejan el lento cambio en la política de producción y venta locales generado por las circunstancias anotadas.

\section{Franco, Mendoza y Gamboa}

La trayectoria literaria de Jorge Franco (Medellín, 1962), Mario Mendoza (Bogotá 1964) y Santiago Gamboa (Bogotá 1965) ha corrido paralela desde sus inicios. Publicaron su primer libro con una diferencia de un año, los tres empezaron a tener notoriedad internacional relativamente rápido, libros de los tres han sido llevados al cine, los tres han recibido un premio literario de peso, los tres han insistido en su carrera literaria publicando con periodicidad, y los tres han sido traducidos. Hay un cierto consenso en considerarlos como la primera avanzada de autores colombianos que accedió al mercado internacional.

La prensa ha sido muy insistente en señalar que son el comienzo de una nueva literatura colombiana: "A partir de sus nombres, de sus obras y de su imagen, las editoriales y los medios de comunicación afirmaron el fin de la era garciamarquiana" (Marín, 2012). Pero esta es una apreciación que está más relacionada con su presencia en los mercados nacionales e internacionales que con su creación literaria. Marín, citando a Gómez (2002), añade: "Sus obras aparecen en los planes lectores de los estudiantes de bachillerato de varios colegios bogotanos... Los estudiantes de Literatura de tres universidades los mencionaron como los escritores colombianos actuales que más conocían o habían leído."

Sin embargo, a la hora de buscar las fuentes de su compartida notoriedad y de analizar las minucias de su carrera literaria las similitudes se acaban. Veremos sus trayectorias editoriales a partir de los elementos constitutivos del prestigio que definimos más arriba.

\section{La construcción del prestigio}

\subsection{Las editoriales}

A partir de la información contenida en worldcat.org, se elaboraron tres cuadros que permiten seguir los cambios de editorial de los autores escogidos. Tal como se puede ver en las tablas 1,2 y $3^{2}$, la relación de los autores escogidos con sus editoriales ha sido cambiante.

El primer libro de Jorge Franco, Maldito amor, fue editado por Ediciones Universidad Central, sede del taller literario más longevo en Bogotá. Cabría recordar que Jorge Franco asistió al Taller Literario de la Biblioteca Pública de Medellín, que dirigía Manuel Mejía Vallejo, y también al taller de la Universidad Central, en donde conoció al escritor Jaime Echevarría, quien fue su maestro. Universidad Central también publicó Mala noche, libro del que hay una edición de JM Calle de Pereira, seguramente parte del premio de Novela Ciudad de Pereira que Mala noche recibiera en 1996. Su tercer libro, Rosario Tijeras (1997), fue editado por Plaza \& Janés en Colombia — sello que esporádicamente editaba escritores colombianos desde finales de la década de 1960 y que en 1984 pasó a formar parte del grupo Bertelsmann. Dice Franco: 
La mandé a Norma y a Planeta. Creo que a Norma la carpeta nunca llegó y en Planeta nunca respondieron. Finalmente la publiqué con Plaza y Janés, donde me dijeron que podían publicar unos 500 ejemplares, pero que no tenían plata para promoción porque iba a salir el nuevo libro de Isabel Allende y le iban a dedicar todo a eso. La novela había ganado la Beca de Creación del Ministerio de Cultura y tenía una plata que me dieron de premio. Ese dinero lo usé en promoción, en hacer unos afiches, en organizar la presentación del libro y en comprar vino para los invitados. Le insistí a la editorial que hiciéramos un contrato, pero no quisieron, dijeron que cuando se acabara la feria del libro hablábamos. (http://www.eltiempo.com/archivo/documento/CMS$\underline{14586656)}$

Cuando la novela empezó a venderse, Norma quiso hacer una edición y también Planeta (con quien firmó contrato por tres libros cuando Gabriel Iriarte era editor). "Comencé la búsqueda de agentes literarios en España y conseguí a Mercedes Casanovas. Ella me dijo 'yo me encargo de eso y tú dedícate a escribir'."

El hecho de que Franco haya buscado un agente a raíz del triunfo de Rosario Tijeras es un gesto indicativo de la profesionalización de los escritores colombianos, y la cita de Franco revela su profunda comprensión de la organización del mercado editorial. El trabajo de Casanovas se notó inmediatamente: empezó a ser publicado por Mondadori en Barcelona, y fue traducido al inglés, al alemán, al polaco, al portugués (a más de quince idiomas). Franco siguió siendo autor de Planeta hasta 2014, cuando recibió el premio Alfaguara de Novela (el último antes de que Alfaguara pasara a formar parte del grupo Random House Mondadori).

Mario Mendoza publicó su primer libro, La ciudad de los umbrales, en Planeta Colombia en 1995, y su editor fue Gabriel Iriarte. En 1998 publicó Scorpio City, en 2001 Relato de un asesino, y en 2002, Satanás. Después de tres novelas publicadas con Planeta, la editorial evidentemente decidió impulsar su candidatura para el premio Seix-Barral, que le fue otorgado ese año a Satanás. Además de Planeta, Mendoza empezó a publicar una saga juvenil en 2013 con la editorial colombiana Arango Editores (el nombre de la serie es El mensajero de Agartha y el primer título fue Mi extraño viaje al mundo de Shambala), pero después del tercer título Planeta asumió la publicación. En 2016, con La melancolía de los feos, Planeta lanzó una colección Mario Mendoza para la cual rediseñó todas las cubiertas de acuerdo con una estética de cartel cinematográfico de película $\mathrm{B}$, que recuerda las cubiertas de las novelas de detectives y de vaqueros que circularon en América Latina en los cincuenta y que eran baratas y populares. La decisión de Mendoza de quedarse en Planeta fue la correcta: el autor colombiano ha sido ampliamente traducido y lo representa en el mercado internacional la agencia de derechos LATAM grupo Planeta ${ }^{3}$.

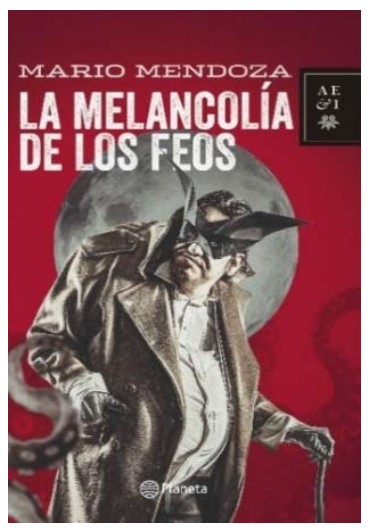

Santiago Gamboa publicó sus primeras dos novelas, Páginas de vuelta (1995) y Perder es cuestión de método (1997) con el Grupo Editorial Norma, y después con la editorial mexicana Grijalbo (que ya en ese momento había sido adquirida por Mondadori) y con Ediciones B (sello español con fílial colombiana que fue adquirido recientemente por Random House). Los impostores (2001) fue publicada por Planeta (bajo el sello Seix-Barral). En 2009 recibió el premio La Otra Orilla, del Grupo Editorial Norma, que se entregaba

\footnotetext{
${ }^{3}$ Cuando Nubia Macías fue directora general de Planeta México implementó la agencia de derechos de Planeta LATAM como una plataforma para fortalecer la oferta de la región desde una gestión diferenciada de la de España (comunicación personal).
} 
por quinta vez y cuya bolsa acababa de aumentar de 30 mil a cien mil dólares. El director editorial de Norma en ese momento era Gabriel Iriarte. El premio se entregó dos veces más (a Gioconda Belli y a Ignacio Padilla) y llegó a su fin con el anuncio del cierre de la editorial a finales de 2010. Al respecto escribió Gamboa en su columna de El Espectador de febrero de 2011:

No es mucho lo que se sabe sobre el cierre de la editorial Norma, pero la noticia no deja de ser, cuando menos, inquietante. Hace tan sólo tres años, con el impulso dado por María Fernanda Carvajal, Norma partió en la dirección contraria: la de treparse a la cumbre de los grandes grupos editoriales en español, es decir Planeta, Santillana y Random House-Mondadori.

Desde 2012 es autor de Random House y su agente es Guillermo Schavelzon, a quien el escritor considera su "consejero espiritual".

\subsection{Los premios}

\section{Jorge Franco}

Primer Premio del Concurso Nacional de Narrativa Pedro Gómez Valderrama por Maldito amor.

Primer Premio del XIV Concurso Nacional de Novela Ciudad de Pereira por Mala noche.

Finalista en el Premio Nacional de Novela de Colcultura con Mala noche.

Beca Nacional de Novela del Ministerio de Cultura con Rosario Tijeras.

2000 Premio Internaciona de Novela Dashiell Hammett por Rosario Tijeras (Gijón, España).

2014 Premio Alfaguara de Novela por El mundo de afuera.

\section{Mario Mendoza}

1995 Premio Nacional de Literatura (área cuento) del Instituto Distrital de Cultura y Turismo de Bogotá por La travesía del vidente.

2002 Premio Biblioteca Breve de Seix Barral por la novela Satanás.

2011 Premio Nacional de Literatura otorgado por la revista Libros y Letras por el conjunto de su obra.

\section{Santiago Gamboa}

2007 Finalista del Premio Rómulo Gallegos con El síndrome de Ulises.

Finalista del Premio Medicis (Francia) a la mejor novela extranjera con la versión francesa de esa novela ("'shortlist").

Finalista del Premio Casino de Póvoa (Portugal) con la traducción portuguesa de la misma obra.

2009 Premio La Otra Orilla por Necrópolis.

2013 Premio Literaturas del Caribe. Mención de honor a Necropolis 1209 (Ed. Métailie).

2014 Premio Coup de Coeur 2014 de Revue Transfuge (París) a Prieres nocturnes (Ed. Métailie).

La trayectoria de premios de los autores considerados es un terreno interesante para analizar las herramientas a disposición de los escritores colombianos. Jorge Franco, en una iniciativa que sin duda se origina en su pertenencia a talleres literarios, empezó recurriendo a los premios locales, que tradicionalmente han servido de apoyo a los escritores colombianos (basta ver, por ejemplo, la trayectoria de Evelio Rosero o de Triunfo Arciniegas): primero el VII Concurso de Historias Cortas Carlos Castro Saavedra, después el Concurso Nacional de Narrativa Pedro Gómez Valderrama, después el Premio Nacional de Novela de Colcultura y por último una beca de creación del Ministerio de Cultura. La función de estos premios es confirmar al escritor en su vocación, conectarlo con el sistema literario local, y en ocasiones permitirle seguir adelante gracias a una bolsa modesta. La presentación de Rosario Tijeras al Premio Internacional de Novela Dashiell Hammett - un premio sin dinero pero creado por un latinoamericano con la expresa intención de abrir el nicho de la novela policiaca en español-, indica la decisión de Franco de saltar a la esfera internacional, y su experiencia editorial hasta ese momento le habría enseñado que no podría contar con un editor para lograrlo. Cuando le dieron el premio Alfaguara, la editorial claramente quería cosechar en un terreno que el autor ya había cultivado, y su efecto tangible fue el cambio de sello del autor. 
El caso de Gamboa es diametralmente básicamente internacional, y no nos permite suponer la intervención de un el sistema editorial, además de una cierta literario en Francia (país donde trabajó premios y menciones de Gamboa habrían ámbito de la alta cultura internacional.

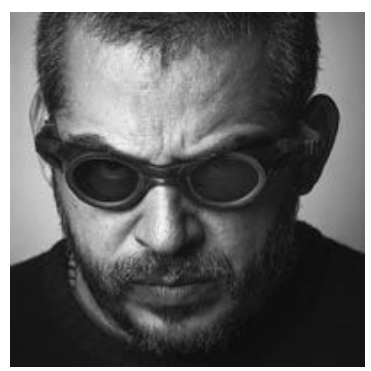

opuesto. Su historial de premios es relacionado con una editorial, lo cual agente (Schavelzon) que conoce bien familiaridad del autor con el sistema varios años como periodista). Los incrementado su prestigio en el Gamboa lo confirma:

Es una vivencia totalmente desconocida para mí, pues es la primera vez en toda mi vida literaria que recibo uno; al principio mandaba cuentos a concursos hasta que desistí por cansancio. Pero sentí que podría pasar porque El síndrome de Ulises fue finalista en el Premio Médicis en Francia a Mejor Libro Traducido; finalista en Portugal y en el Rómulo Gallegos cuando ganó Elena Poniatowska. Cuando mi agente literario, que es como un consejero espiritual, propuso que lo mandáramos a un premio importante, me alegré. Para mí los premios son reconocimientos de colegas a un colega, financiado por las editoriales, así lo miro. En este caso es una gran emoción que lo hayan hecho Jorge Volpi, Roberto Ampuero y Pere Sureda, encargado en la editorial en España, un gran intelectual que conozco hace años. ([http://tanialu.co/2009/09/19/necropolis-desantiago-gamboa-recibe-el-premio-la-otra-orilla/\#sthash.lx6b4TGV.5Y2XCUVJ.dpbs]

Mendoza empezó su carrera con el Premio Nacional de Literatura (área cuento) del Instituto Distrital de Cultura y Turismo de Bogotá por La travesía del vidente, un premio que ha marcado el comienzo de muchos escritores colombianos. El siguiente premio, el Seix Barral, es un premio de la casa editorial de Mendoza, y tendría sentido como parte de una estrategia para internacionalizar al autor. La brutal reacción de la crítica en España hubiera podido dar al traste con la carrera de Mendoza, quien escogió a partir de ese momento un rumbo más acorde con sus inclinaciones literarias y menos volcado al aval de la crítica.

En 2011, Mendoza recibió el VIII Premio Nacional de Literatura otorgado por la fundación Cultural Libros \& Letras, un premio que se asigna con los votos de los lectores. En una entrevista con un periódico barrial, Mendoza afirmó lo siguiente: "Es el mejor premio que he recibido, por encima del Seix Barral que me entregaron en España y el del Instituto de Cultura, este lo entregan los lectores y para mí tiene un significado más especial".

\subsection{La prensa}

Se hizo una revisión de los artículos de prensa aparecidos entre 2002 y 2017 (accesibles en la red) que se ocuparan de los autores escogidos. Lo primero que salta a la vista es que la mayor parte de los artículos son de índole noticiosa, no son reseñas ni comentarios críticos. En la prensa aparece como noticia los movimientos de los escritores, las nuevas publicaciones, los premios. Este último tema es el que ocupa la mayor parte de los artículos y nos lleva a la conclusión de que el aporte primordial de la prensa tiene que ver con el reconocimiento del escritor como figura pública. El género preferido por la prensa para dar cuenta de las noticias literarias, además de la breve reseña del evento, es la entrevista. Resulta relevante constatar que los autores aprenden a utilizar las entrevistas para moldear su manera particular de ocupar una posición objetiva en el campo literario, para negociar su forma personal de habitar el rol del escritor, su postura. Al respecto dice Meizoz (2): "J'ai défini la posture comme la présentation de soi d'un écrivain, tant dans sa gestion du discours que dans ses conduites litttéraires publiques. Le meilleur équivalent à cette notion serait le terme latin de persona qui désigne le masque de théàtre".

El caso de Mendoza es el más interesante. Ya hablamos del reciente cambio de portadas en Planeta, y habría que añadir la imagen del autor que ilustra la página de la editorial, una fotografía que alude a lo posapocalíptico, que hace referencia a los contenidos de su obra que Mendoza quiere subrayar. En un artículo en la revista Semana, el autor habla de la posibilidad de que su obra "quizás ha dado en el blanco de una época turbia, siniestra y sumamente cruel" en la que "la enorme mayoría caminamos por una cornisa, haciendo equilibrio para no caernos en un agujero negro, al borde de nuestros propios precipicios". 
Mendoza se define a sí mismo como un hombre que viene de la academia, y hasta allí rastrea sus orígenes:

Tengo dos influencias marcadas... mi padre, un profesor jubilado de la Universidad Nacional, que ejerció allí su profesión como profesor de Veterinaria durante 35 años, tenía una biblioteca estupenda, hablaba cuatro idiomas y era un representante de esa clase media ilustrada latinoamericana que valora la educación por encima de cualquier cosa... Y un profesor de literatura extraordinario: Eduardo Jaramillo, quien más adelante sería un crítico muy reconocido en Estados Unidos, además de destacado profesor universitario... En el año 2000 soy profesor de Literatura en la Universidad Javeriana tanto del Pregrado como de la Maestría y tengo una crisis que me hace jugármela por el mundo de la escritura todo o nada. (2014 comunicado del Ministerio de Cultura)

Desde su experiencia en el salón de clase, Mendoza conecta con sus estudiantes y va formando un grupo de lectores jóvenes, un grupo de lectores cada vez más numeroso a los que Mendoza atiende y con los que quiere relacionarse personalmente. Cuando lanzó su novela Apocalipsis, "el novelista quiso premiar a sus lectores y abrió una convocatoria de textos sobre su obra para que los autores de los tres mejores presentaran el libro. Una bacterióloga, una estudiante de diseño de séptimo semestre y un sociólogo que trabaja como profesor de literatura en un colegio de Sibaté fueron los elegidos. La carátula del libro también fue escogida por concurso y lo ganaron los jóvenes Mauricio Gaitán y Freddy Ospina." (Arcadia, 2011)

Santiago Gamboa, compañero de Mendoza en el colegio Refous y en la Universidad Javeriana, ha optado por definirse como un autor más literario, con raíces en el periodismo, desde donde pasó a la literatura: “¿Cuántos lectores tienes?”, le preguntan en una entrevista para El mundo de España. "Menos de los que tenía cuando era periodista." Desde allí, desde la columna de opinión que ha conservado casi siempre a lo largo de su carrera, Gamboa se perfila a sí mismo más como el intelectual público que como el escritor de masas. En reportaje publicado por periódico chileno La tercera en 2012, se afirma que:

el escritor dice tener la sensación de que "la literatura dura y pura fue víctima de un golpe de Estado de otros tipos de libro, el libro de entretenimiento", indicó el autor de Perder es cuestión de método, Octubre en Pekín o Los impostores. Para Gamboa "el autor de libros de entretenimiento no tiene una intención artística en su trabajo" y denunció que es quien "ha suplantado a la figura del escritor de literatura en muchos espacios". Tanto en Colombia "como en España o en Estados Unidos, los escritores están sumergidos en ese golpe de Estado", reiteró Gamboa, quien, por otra parte, se declaró halagado por la coincidencia expresada de ese temor que hizo el escritor peruano Mario Vargas Llosa.

Unos años más tarde, a raíz de la publicación La guerra y la paz, responde lo siguiente a la pregunta sobre su incursión en el ensayo:

Toda esa parte de ejecutar mi propia educación, mis intereses, la sacaba por la vía de las columnas de opinión. Escribo con regularidad en varios periódicos de América Latina. Pero, en este caso, de repente sentí que todo se ordenaba en torno a la idea que me ha preocupado en la literatura, en mi propia vida, en lo que le pasa a mi país. Yo no vivo físicamente en Colombia, pero soy colombiano; leo todos los días, antes que muchos colombianos, los periódicos, oigo la radio, me mantengo informado sobre el país. (http://www.eltiempo.com/archivo/documento/CMS-13994776)

Jorge Franco es el único de los tres que se define a sí mismo exclusivamente como un escritor (ni periodista, ni académico, ni intelectual), como lo demuestra su trayectoria editorial (ver 5.1). Sus declaraciones, sus reportajes, hablan del oficio, de las herramientas de la escritura, de las dificultades o 
logros en la construcción de sus novelas. Sus anécdotas se refieren a momentos relacionados con la escritura, y se dibuja constantemente como un escritor (la vida familiar en relación con las rutinas de trabajo, las noticias relevantes de su vida en relación con el oficio).

$\mathrm{Su}$ relación con sus lectores es distante, abstracta. Ante la pregunta sobre "la respuesta de la gente ante Rosario Tijeras 15 años después de haberse publicado", Franco responde:

Es una novela que sigue completamente viva, la siguen leyendo mucho en los colegios, siempre en mi agenda visito instituciones y me agrada mucho saber que ellos mismos son quienes piden la lectura y eso me parece maravilloso porque aporta un grano de arena a cultivar el hábito de la lectura, que es algo muy difícil de lograr entre los muchachos.

\subsection{La academia}

En 2002, en un artículo de la revista Semana, el crítico David Jiménez resumió la posición de la academia frente a escritores como Franco, Mendoza o Gamboa: "Uno de los graves problemas para esta nueva generación es la falta de crítica y de personas que realmente los pongan en su lugar", dijo:

Esta generación ha surgido en la opinión de publicistas del periodismo. Sean buenos, regulares o malos escritores, nadie los ha puesto en su lugar. Pero no es culpa de ellos. Los medios son los que les han hecho publicidad a sus libros pero nadie se ha atrevido a cuestionarlos. Aun así y aunque yo tenga mi opinión sobre algunas obras es muy difícil presagiar quién será un gran autor cuando aún no pasa de los 30 años. Como hay otros que a los 50 ya mostraron todo su talento.

Diez años después, la ensayista Consuelo Triviño (2012) lleva la opinión de Jiménez un poco más lejos:

La década de los noventa se caracteriza por la presencia de los nacidos a partir de los sesenta que, como he dicho, pretenden desmarcarse de una tradición literaria que consideran "cargada de referencias librescas" y escrita para un público "culto". Apoyados por los poderosos grupos editoriales se les ha dado a conocer en circuitos internacionales y se les han otorgado los más prestigiosos premios y, además, en muchos casos, las bibliotecas públicas del país que adquieren sus libros favorecen con esa "ayuda" a las editoriales y, a la vez, los promocionan. Estos declaran no tener prejuicios estéticos y dirigirse un lector "menos snob", como diría en una entrevista Efraim Medina Reyes (Cartagena, 1967).

No obstante, la academia ha acabado cediendo a la evidencia de la voz de esta nueva generación de escritores, y en apariencia ha optado por rescatarlos para las aulas como representantes del género negro. Así lo confirman los textos escritos por Andrea Fanta (2012), por ejemplo, o la compilación de ensayos sobre el tema de Craig-Odders, Collins y Close (2006). En el dossier sobre género negro que la revista Hojas universitarias publicó en abril de 2008, ya no se habla de esta generación como herederos de García Márquez sino como herederos de Raymond Chandler. En el manual de novela colombiana de Jaime Alejandro Rodríguez Ruiz, de la Universidad Javeriana, también aparecen como representantes de la novela policiaca (en http://www.javeriana.edu.co/narrativa_colombiana/contenido/bibliograf/pineda_mito/introduccion.htm)

\section{Conclusiones}

La caracterización de las trayectorias editoriales de Mario Mendoza, Jorge Franco y Santiago Gamboa nos permitió determinar algunas de las fuentes y los modos en los que los narradores colombianos contemporáneos construyen su prestigio literario. Su aparición en el campo literario en momentos en que las grandes editoriales españolas que dominaban el mercado literario colombiano optaron por publicar masivamente a los autores locales les permitió acceder rápidamente a los mercados internacionales, guiados, en el caso de Franco y de Gamboa, por sus agentes, y en el caso de Mendoza, por su editorial. Los tres han demostrado a lo largo de sus carreras que entienden el campo literario y que saben moverse en él para consolidar su prestigio. Así lo demuestran sus decisiones editoriales y el hecho de que hayan buscado un agente; la publicación periódica de novedades y su presencia constante en las noticias indican que los tres se conciben como escritores profesionales, cada uno con una persona pública reconocible que han construido cuidadosamente. 


\section{Tabla 1 - Organización de las obras de Jorge Franco Ramos por fecha de publicación y por editorial}

\begin{tabular}{|l|l|l|l|}
\hline \multicolumn{3}{|c}{ ORGE FRANCO RAMOS } \\
\hline Franco & Mala noche & Ed Universidad Central & 1996 \\
\hline Franco & Mala noche & JM Calle - Pereira & 1997 \\
\hline Franco & Mala noche & Plaza y Janés & 1997 \\
\hline Franco & Rosario Tijeras & Plaza y Janés & 1997 \\
\hline Franco & Rosario Tijeras & Planeta & 1999 \\
\hline Franco & Rosario Tijeras & Plaza y Janés & 1999 \\
\hline Franco & Paraíso Travel & Planeta & 2001 \\
\hline Franco & Paraíso Travel & Quebecor World & 2001 \\
\hline Franco & Mala noche & Planeta & 2003 \\
\hline Franco & $\begin{array}{l}\text { Donde se cuenta como me encontre } \\
\text { con Don Quijote de la Mancha en }\end{array}$ & Planeta & 2005 \\
& $\begin{array}{l}\text { Medellín, cuando la ciudad se lleno de } \\
\text { gigantes inventados }\end{array}$ & & \\
\hline Franco & Melodrama & Mondadori & \\
\hline Franco & Melodrama & Planeta & 2006 \\
\hline Franco & Melodrama & Planeta & 2006 \\
\hline Franco & Santa suerte & 2006 \\
\hline Franco & Santa suerte & Planeta & 2010 \\
\hline Franco & Santa suerte & Alfaguara & 2012 \\
\hline Franco & Santa suerte & Planeta & 2012 \\
\hline Franco & El mundo de afuera & Seix Barral & 2012 \\
\hline Franco & El mundo de afuera & Alfaguara & 2014 \\
\hline Franco & La niña calva & Santillana & 2014 \\
\hline & & Alfaguara & 2014 \\
\hline
\end{tabular}

FUENTE: Worldcat.org -

https:/www.worldcat.org/profiles/mvalenciav/lists/3782888/bibliography?view=\&style= APA\&export=HTML\&se=as\&sd=asc\&qt=sort_as_asc 
Tabla 2 - Organización de las obras de Santiago Gamboa por fecha de publicación y por editorial

\begin{tabular}{|c|c|c|c|}
\hline \multicolumn{4}{|c|}{ SANTIAGO GAMBOA } \\
\hline Gamboa & Páginas de vuelta & Norma & 1995 \\
\hline Gamboa & Perder es cuestión de método & Grijalbo & 1997 \\
\hline Gamboa & Perder es cuestión de método & Mondadori & 1997 \\
\hline Gamboa & Perder es cuestión de método & Norma & 1997 \\
\hline Gamboa & Páginas de vuelta & Mondadori & 1998 \\
\hline Gamboa & Páginas de vuelta & Mondadori & 1998 \\
\hline Gamboa & Páginas de vuelta & Norma & 1998 \\
\hline Gamboa & $\begin{array}{l}\text { Vida feliz de un joven llamado } \\
\text { Esteban }\end{array}$ & Barcelona - Suma de Letras & 2000 \\
\hline Gamboa & $\begin{array}{l}\text { Vida feliz de un joven llamado } \\
\text { Esteban }\end{array}$ & Ediciones B & 2000 \\
\hline Gamboa & Octubre en Pekin & Mondadori & 2001 \\
\hline Gamboa & Octubre en Pekin & Mondadori & 2001 \\
\hline Gamboa & Páginas de vuelta & Mondadori & 2001 \\
\hline Gamboa & Les captifs du lys blanc & Paris - Métailie & 2002 \\
\hline Gamboa & Los impostores & Planeta & 2002 \\
\hline Gamboa & Los impostores & Seix Barral & 2002 \\
\hline Gamboa & Octubre en Pekin & Mondadori & 2002 \\
\hline Gamboa & Ottobre a Pechino & U.Guanda & 2002 \\
\hline Gamboa & El síndrome de Ulises & Planeta & 2005 \\
\hline Gamboa & El síndrome de Ulises & Planeta & 2005 \\
\hline Gamboa & El síndrome de Ulises & Seix Barral & 2005 \\
\hline Gamboa & El síndrome de Ulises & Seix Barral & 2005 \\
\hline Gamboa & Hotel Pekin & Seix Barral & 2005 \\
\hline Gamboa & Hotel Pekin & Planeta & 2008 \\
\hline Gamboa & Necrópolis & La otra orilla & 2009 \\
\hline Gamboa & Necrópolis & Norma & 2009 \\
\hline Gamboa & Necrópolis & Norma & 2009 \\
\hline Gamboa & Nekropolis & Brno - Host & 2009 \\
\hline Gamboa & Hotel Pekin & $\begin{array}{l}\text { Warzaw:Warzawskie } \\
\text { Literackie }\end{array}$ & 2010 \\
\hline Gamboa & Octubre en Pekin & Mondadori & 2012 \\
\hline Gamboa & Plegarias nocturnas & Mondadori & 2012 \\
\hline Gamboa & $\begin{array}{l}\text { Océanos de arena: Diario de } \\
\text { viaje por Medio Oriente }\end{array}$ & Mondadori & 2013 \\
\hline Gamboa & Plegarias nocturnas & Mondadori & 2013 \\
\hline Gamboa & La guerra y la paz & Debate & 2014 \\
\hline Gamboa & La guerra y la paz & Penguin Random House & 2014 \\
\hline Gamboa & $\begin{array}{l}\text { Océanos de arena: Diario de } \\
\text { viaje por Medio Oriente }\end{array}$ & Random House & 2014 \\
\hline Gamboa & Una casa en Bogotá & Penguin Random House & 2014 \\
\hline Gamboa & Una casa a Bogotá & Edizioni e/o & 2016 \\
\hline Gamboa & Volver al oscuro valle & Penguin Random House & 2016 \\
\hline Gamboa & Octubre en Pekin & Random House & 2017 \\
\hline Gamboa & Return to the Dark Valley & La Vergne - Europe Editions & 2017 \\
\hline
\end{tabular}

FUENTE: Worldcat.org -

https://www.worldcat.org/profiles/mvalenciav/lists/3782888/bibliography?view=\&st yle=APA\&export=HTML\&se=as\&sd=asc\&qt=sort_as_asc 


\section{Tabla 3 - Organización de las obras de Mario Mendoza por fecha de publicación y por editorial}

\begin{tabular}{|c|c|c|c|}
\hline \multicolumn{4}{|c|}{ MARIO MENDOZA } \\
\hline Mendoza & Relato de un asesino & Planeta & 2001 \\
\hline Mendoza & Satana & Torino - Einaudi & 2002 \\
\hline Mendoza & Satanás & Planeta & 2002 \\
\hline Mendoza & Satanás & Seix Barral & 2002 \\
\hline Mendoza & El viaje del loco Tafur & Seix Barral & 2003 \\
\hline Mendoza & Relato de un asesino & Seix Barral & 2003 \\
\hline Mendoza & Relato de un asesino & Planeta & 2004 \\
\hline Mendoza & Relato de un asesino & Seix Barral & 2004 \\
\hline Mendoza & Scorpio city & Planeta & 2004 \\
\hline Mendoza & Scorpio city & Seix Barral & 2005 \\
\hline Mendoza & Scorpio city & Planeta & 2006 \\
\hline Mendoza & Los hombres invisibles & Planeta & 2007 \\
\hline Mendoza & Los hombres invisibles & Seix Barral & 2007 \\
\hline Mendoza & Scorpio city & Planeta & 2008 \\
\hline Mendoza & Buda blues & Planeta & 2009 \\
\hline Mendoza & Scorpio city & Planeta & 2009 \\
\hline Mendoza & Buda blues & Seix Barral & 2010 \\
\hline Mendoza & La locura de nuestro tiempo & Planeta & 2010 \\
\hline Mendoza & La locura de nuestro tiempo & Seix Barral & 2010 \\
\hline Mendoza & Scorpio city & Seix Barral & 2010 \\
\hline Mendoza & Apocalipsis & Planeta & 2011 \\
\hline Mendoza & Apocalipsis & Planeta & 2012 \\
\hline Mendoza & La importancia de morir a tiempo & Planeta & 2012 \\
\hline Mendoza & La importancia de morir a tiempo & Planeta & 2013 \\
\hline Mendoza & La locura de nuestro tiempo & Planeta & 2013 \\
\hline Mendoza & Lady Masacre & Planeta & 2013 \\
\hline Mendoza & Mi extraño viaje al mundo de Shambala & Arango Editores & 2013 \\
\hline Mendoza & Apocalipsis & Planeta & 2014 \\
\hline Mendoza & Paranormal Colombia (al filo de lo real) & Planeta & 2014 \\
\hline Mendoza & Scorpio city & Planeta & 2014 \\
\hline Mendoza & La locura de nuestro tiempo & Planeta & 2015 \\
\hline Mendoza & La melancolía de los feos & Planeta & 2016 \\
\hline Mendoza & Lady Masacre & Destino & 2016 \\
\hline
\end{tabular}

FUENTE: Worldcat.org -

https://www.worldcat.org/profiles/mvalenciav/lists/3782888/bibliography?view=\&styl $\mathrm{e}=\mathrm{APA} \& \operatorname{export}=\mathrm{HTML} \& \mathrm{se}=\mathrm{as} \& \mathrm{sd}=\mathrm{asc} \& \mathrm{qt}=$ sort_as_asc 


\section{Referencias bibliográficas}

Anderson, Danny (1996), “Creating Cultural Prestige: Editorial Joaquín Mortiz”, Latin American Reaearch Review, vol. 31 (2).

Bourdieu, Pierre (1997). Las reglas del arte; génesis y estructura del campo literario. Barcelona: Anagrama.

Comunicado del Ministerio de Cultura (2014). Mendoza en la Feria itinerante de libreros independientes de Riohacha

Craig-Odders, Renée W., Jacky Collins y Glen S. Close (2006). Hispanic and Luso-brazilian Detective Fiction: Essays on the Genero Negro Tradition. Jefferson, N.C.: McFarland \& Company.

Curtius, E.R. (1953). European Literature and the Latin Middle Ages. Princeton: Princeton University Press.

De Glas, Frank (1998), “Authors' oeuvres as the backbone of publishers' lists: Studying the literary publishing house after Bourdieu", Poetics 25 (1998), pp. 379-397.

Dubois, Jacques (2014). La institución de la literatura. Medellín: Universidad de Antioquia, 2014

Fanta, Andrea (2012), "Perder es cuestión de método y las paranarraciones de Santiago Gamboa", Boletín Hispánico Helvético, volumen 20, otoño 2012, pp. 21-39

Fernández Moya, María (2009), "Multinacionales del castellano. El proceso de internacionalización del sector editorial español 1898-2008”, Revista de Historia Industrial 40, año XVIII, 2, 2009, p. 43.

Herrera-Olaizola, Alejandro (2000), "Consuming Aesthetics", MLN volumen 115 \#2 March, 2000.

Jackson, H.L. (2015). Those Who Write for Immortality. London: Yale University Press.

Marín, Maribel (2017), “AA quién sirven los premios literarios?”, Babelia, 13 de febrero de 2017. Disponible en: elpais.com/cultura/2017/02/10/babelia/1486723630 593071. Capturado el 25 de marzo de 2017.

Marín, P.A. (2012), "La novela colombiana reciente ante el mercado: críticos contra lectores", Literatura, teoría historia y crítica vol 14 (1), enero-junio.

Meizoz, Jérôme (2009), “Ce que l'on faire dire au silence: posture, ethos, image d'autheur”, Argumentation et Analyse de Discours 3/2009.

Disponible en: https://journals.openedition.org/aad/667

"Necrópolis de Santiago Gamboa recibe el premio La otra orilla" (2009). Disponible en: tanialu.co/2009/09/19/necropolis-de-santiago-gamboa-recibe-el-premio-la-otraorilla/\#sthash.lx6b4TGV.5Y2XCUVJ.dpbs, 2009.

Pera, Cristóbal (2015), “The Promise of Spanish Publishing in Mexico and the United States", Pub Res Q (2015) 31:7379.

Triviño, Consuelo (2012), “La narrativa colombiana ante el marketing 1992-2012”, Omnibus 40.

Disponible en: www.omnibus.com/n40/sites.google.com/site/omnibusrevistainterculturaln40/articulos/narrativa/lanarrativa-colombiana-ante-el-marketing-1992-2012

Underwood, Ted y Jordan Sellers (2016), “The Long Durée of Literary Prestige”, Modern Language Quarterly 77:3.

Vanderbilt, Tom (2012), "Why is Literary Fame so Unpredictable?”, The New Yorker, May 21. Disponible en: https://www.newyorker.com/books/page-turner/why-is-literary-fame-so-unpredictable?

Verboord, Marc (2003), "Classification of Authors by Literary Prestige”, Poetics 31, pp. 259-281.

Verboord, Marc (2011), "Market logic and Cultural Consecration in French, German, and American bestseller lists 1970-2007”, Poetics 39, pp. 290-315. 